Abstract 05-S2.04 Table 1 Sexually transmitted infections provider knowledge, beliefs and attitudes in Gauteng, South Africa, 2008-2009

\begin{tabular}{|c|c|c|c|c|c|c|}
\hline \multirow[b]{2}{*}{$\mathrm{N}=611$} & \multicolumn{2}{|c|}{ Agree } & \multicolumn{2}{|c|}{ Disagree } & \multicolumn{2}{|c|}{ Do Not Know } \\
\hline & $\%$ & $\mathbf{n}$ & $\%$ & $\mathbf{n}$ & $\%$ & $\mathbf{n}$ \\
\hline \multicolumn{7}{|l|}{ Knowledge } \\
\hline Some STIs cannot be cured with medication & 48.1 & 294 & 45.7 & 279 & 6.2 & 38 \\
\hline Genital herpes often recurs & 93.1 & 569 & 3.8 & 23 & 3.1 & 19 \\
\hline $\begin{array}{l}\text { Genital Herpes often causes urethral } \\
\text { discharge in men }\end{array}$ & 25.7 & 157 & 87.4 & 377 & 12.6 & 77 \\
\hline Genital herpes is curable & 42.2 & 258 & 4.8 & 324 & 53.0 & 29 \\
\hline $\begin{array}{l}\text { Genital Herpes sores can be treated with } \\
\text { medication(s) }\end{array}$ & 84.9 & 519 & 10.0 & 61 & 5.1 & 31 \\
\hline Untreated STIs can develop into AIDS & 33.9 & 207 & 60.6 & 370 & 5.6 & 34 \\
\hline $\begin{array}{l}\text { Many patients with STIs already have HIV } \\
\text { or AIDS }\end{array}$ & 42.4 & 259 & 52.2 & 319 & 5.4 & 33 \\
\hline \multicolumn{7}{|l|}{ Beliefs } \\
\hline $\begin{array}{l}\text { I think one of my most important respon- } \\
\text { sibilities is to strongly recommend HIV } \\
\text { testing to each of my STI patients }\end{array}$ & 93.6 & 572 & 5.1 & 31 & 1.3 & 8 \\
\hline $\begin{array}{l}\text { I think traditional herbal medicine is able to } \\
\text { cure some STIs }\end{array}$ & 16.2 & 99 & 81.0 & 495 & 2.8 & 17 \\
\hline $\begin{array}{l}\text { I think traditional herbal medicine is able to } \\
\text { cure HIV/AIDS }\end{array}$ & 1.5 & 9 & 79.7 & 487 & 18.8 & 115 \\
\hline $\begin{array}{l}\text { In the long run, some HIV medication(s) can } \\
\text { be more dangerous than having AIDS }\end{array}$ & 31.1 & 190 & 65.1 & 398 & 3.8 & 23 \\
\hline \multicolumn{7}{|l|}{ Attitudes } \\
\hline $\begin{array}{l}\text { Under certain circumstances, it is OK to test } \\
\text { patients for HIV without telling them }\end{array}$ & 26.0 & 159 & 71.8 & 439 & 2.1 & 13 \\
\hline
\end{tabular}

\section{5-S2.05 ROLE OF REGIONAL REFERENCE LABORATORIES FOR SEXUALLY TRANSMITTED INFECTIONS IN IMPROVEMENT OF SECOND GENERATION HIV SURVEILLANCE EXPERIENCE FROM THE CENTRAL AMERICA REGION}

doi:10.1136/sextrans-2011-050109.161

T Ye, L Steele, S I Juarez, C Y Chen, R C Ballard. Centers for Disease Control and Prevention Atlanta, USA

Background WHO/UNAIDS recommends National HIV/AIDS and Sexually Transmitted Infection STI) Control Programs to implement integrated HIV, STI and behavioural surveillance to assess the disease burden, and to monitor the temporal trends of HIV/STI prevalence and high-risk sexual behaviours. Despite this recommendation, there is limited STI prevalence data available in many resource poor countries owing to lack of STI laboratory capacity to detect multiple STIs. The CDC STD lab explored the feasibility of a decentralised laboratory strengthening approach by applying more advanced molecular STI diagnostic methods to support the second generation surveillance (SGS) activities in the Central American Region during 2007-2011.

Methods CDC STD lab developed a real-time multiplex PCR (MPCR) to detect Neisseria gonorrhoeae, Chlamydia trachomatis, Trichomonas vaginalis, and Mycoplasma genitalium. In collaboration with regional partners, CDC identified and transferred the diagnostic technology to a regional STI reference laboratory in 2008. The regional reference laboratory provides MPCR testing on specimens collected for surveillance purpose. This standardised SGS study design was implemented at regular intervals in multiple countries in the region to estimate the disease burden and to monitor temporal STI trends.

Results During 2007-2010, four national integrated sexual behavioural and biomarker surveys were implemented using CDC's affiliated regional STI reference center. The quality STI prevalence data was made available to the national HIV/STI programs in Honduras, El Salvador, Nicaragua and Colombia. Similar CDC supported surveillance studies are in-preparation in Guatemala, Belize, Panama, Honduras (second round) and the Dominican Republic.
Conclusions Based on the experience from the Central American Region, it is feasible to implement integrated HIV, STI and behavioural surveillance surveys using robust molecular techniques in resource poor settings. In collaboration with regional partners, CDC's approach to STI laboratory capacity strengthening through establishing a regional reference laboratory should be expanded to other regions.

\section{5-S2.06 ABSTRACT WITHDRAWN}

\section{Health services and policy oral session 3 - partner notification \\ 05-S3.01 USE AND EFFECTIVENESS OF EXPEDITED PARTNER THERAPY IN AN INNER-CITY STD CLINIC}

doi:10.1136/sextrans-2011-050109.163

T Mickiewicz, A Al-Tayyib, C Mettenbrink, C Rietmeijer. Denver Public Health, Denver, USA

Background In November 2006, the Denver Metro Health (STD) Clinic (DMHC) began offering Expedited Partner Therapy EPT) to heterosexual patients infected with Chlamydia trachomatis $(\mathrm{Ct})$ or Neisseria gonorrhoeae (GC). Among those who accept EPT, the patient delivers treatment to his or her partner(s), removing the need for a clinic visit. We investigate demographic differences among patients who accepted EPT and examine re-infection rates among those who return to the clinic.

Methods Data were extracted from the electronic medical record (EMR) for 2644 eligible patients offered EPT between November 2006 and October 2010. Acceptance rates are compared across demographics and infection status. Rates of re-infection are examined among the 339 patients who returned for re-testing within 21 to 90 days of treatment. Dual infections are excluded. Results Overall, 763 (28.3\%) eligible patients accepted EPT. Women were more likely than men to accept EPT ( $35.9 \%$ vs $23.8 \%$, p $<0.01)$. Patients younger than 40 accepted EPT at a higher rate than those 40 or older $(29.6 \%$ vs $20.0 \%, p<0.01)$. African Americans were least likely to accept EPT (23.0\%), whereas approximately $30 \%$ of Hispanics and Whites accepted and those reporting multiple races accepted at the highest rate $(43.6 \%)(p<0.01)$. Ct infected patients were significantly more likely to accept EPT than those infected with GC $(32.7 \%$ vs $24.6 \%, \mathrm{p}<0.01)$. Within 21 to 90 days of treatment initiation, 339 patients returned for re-testing (221 for $\mathrm{Ct}$ and 118 for GC). Overall, re-infection rates differed significantly by EPT acceptance at the initial visit with $11.1 \%$ re-testing positive among those who accepted EPT compared to $20.4 \%$ among those who refused $(p=0.04)$ (Abstract O5-S3.01 table 1$)$. When stratified by type of infection, the differences were no longer significant but the direction of the relationship remained. Among those originally $\mathrm{Ct}$ infected, $9.1 \%$ of those who accepted EPT re-tested positive vs $15.3 \%$ among those who refused $(p=0.19)$. Among those originally GCinfected, $16.1 \%$ of EPT acceptors re-tested positive compared to $28.7 \%$ of those who refused $(p=0.17)$.

Abstract 05-S3.01 Table 1 Use and effectiveness of expedited partner therapy in an inner-city STD clinic Mickiewicz T, Al-Tayyib AA, Mettenbrink C, Rietmeijer CA

\begin{tabular}{llll}
\hline \multicolumn{4}{l}{ Re-infection rates among those returning to clinic for re-testing } \\
\hline Ct & GC & Ct or GC* \\
\hline Accepted EPT & $7 / 77(9.1 \%)$ & $5 / 31(16.1 \%)$ & $12 / 108(11.1 \%)$ \\
Refused EPT & $22 / 144(15.3 \%)$ & $25 / 87(28.7 \%)$ & $47 / 231(20.4 \%)$ \\
\hline
\end{tabular}

${ }^{*} p<0.05$. 
Conclusions In a busy inner-city STD clinic, EPT is more likely to be accepted by women, those who are younger and those infected with $\mathrm{Ct}$. Re-infection rates among patients returning to the clinic suggest that EPT reduces the risk of re-infection, with the greatest benefit among those originally infected with GC.

\section{5-S3.02 IMPLEMENTATION OF AN EXPEDITED PARTNER THERAPY (EPT) PROGRAM IN AN INNER-CITY STD CLINIC}

doi:10.1136/sextrans-2011-050109.164

T Mickiewicz, A Al-Tayyib, C Mettenbrink, C Rietmeijer. Denver Public Health Denver, USA

Background Expedited partner therapy (EPT) is the practice of treating sex partners of persons with sexually transmitted diseases (STD) without an intervening medical evaluation. In 2006, the CDC issued guidelines for providing EPT to heterosexual patients diagnosed with gonorrhoea/chlamydial infection, allowing them to deliver treatment to their partner(s). In November 2006, the Denver Metro Health (STD) Clinic (DMHC) began offering EPT. We describe EPT implementation challenges and process improvements. Methods Three phases of implementation are described: 1) the pilot phase, 2) implementation of a quality assurance protocol and 3) initiation of a prompt in the electronic medical record (EMR). Data were extracted from the EMR to calculate acceptance rates of EPT among the 2056 eligible patients over this time period. Rates were examined by month and for each phase.

Results Before initiation of the pilot phase, a protocol to dispense EPT was developed and staff trained. During the pilot phase (September 2007-April 2008), patient acceptance rates averaged 17\% (range: $8 \%-22 \%$ ). As rates were not improving, EMR data were examined to determine potential areas for intervention. At DHMC, clinical services are provided by health care partners (HCP) and licensed nurse practitioners (LNP). Analyses revealed significant differences in patient acceptance of EPT by provider type: HCP providers were less likely to have patients accept EPT than LNP providers (prevalence ratio $=1.7,95 \% \mathrm{CI}: 1.5 \%$ to $1.9 \%$ ), likely due to the nature of the visit and the volume of patients seen by HCP providers. Targeted re-training was initiated and quality assurance reports were generated monthly. Although the acceptance rate increased significantly to $22 \%(p=0.04)$, there was no indication that rates were increasing with time. In January 2009, the EMR was amended, requiring all providers to document EPT referral, accept- ance or refusal (and reason for refusal), before closing the patient EMR. Rates improved significantly to an overall rate of $49 \%$ $(p<0.01)$ through October 2010 (Abstract O5-S3.02 figure 1) Reasons for refusal among those who did not accept EPT included that partner would be notified (42.5\%), partner was being treated $(30.1 \%)$ or the patient was not able to contact the partner $(21.9 \%)$ Conclusions In a busy inner-city STD clinic, an automated EMR prompt that forces documentation of EPT provided the greatest success. An EPT acceptance rate of 50\% may be the optimum rate in a real-world setting.

\section{5-S3.03 INCREMENTAL COST-EFFECTIVENESS OF INTRODUCING PARTNER NOTIFICATION WITH SELECTIVE SCREENING FOR STD CONTROL IN LOUISIANA}

doi:10.1136/sextrans-2011-050109.165

${ }^{1} \mathrm{M}$ Rahman, ${ }^{2} \mathrm{M}$ Khan, 'L Longfellow. 'Louisiana Office of Public Health, Metairie, USA; ${ }^{2}$ Tulane University, New Orleans, USA

Background Selective screening and partner notification are the two strategies used by the STD control programs in USA to reduce and or eliminate syphilis. So far no study has assessed the cost and effectiveness of either approach at the state level. The objective of this study is to assess the incremental cost effectiveness (ICE) of adding partner notification with selective screening (SS) in detecting early syphilis and to measure ICE of intensity of partner notification in Louisiana.

Method The cost effectiveness analysis (CEA) was done from the point of view of health care delivery. Micro costing approach was used in cost analysis and the CEA was performed by using the recurrent direct costs associated with detecting syphilis by SS and by SS with partner notification see Abstract O5-S3.03 figure 1. For ICE of intensity of partner notification, cost was calculated for every attempt made to contact a partner and effectiveness was calculated by the number of partners identified as well as the number of cases identified through partner notification.

Results The estimates of direct costs associated with SS was $\$ 6.4$ million for 1005 early syphilis cases detected and \$6.7 million for SS with partner notification. Partner notification detected additional 279 early syphilis cases with an additional cost of $\$ 314498$. Incremental cost of adding partner notification with SS was $\$ 2808$ per primary and secondary syphilis case, $\$ 1883$ per early latent syphilis

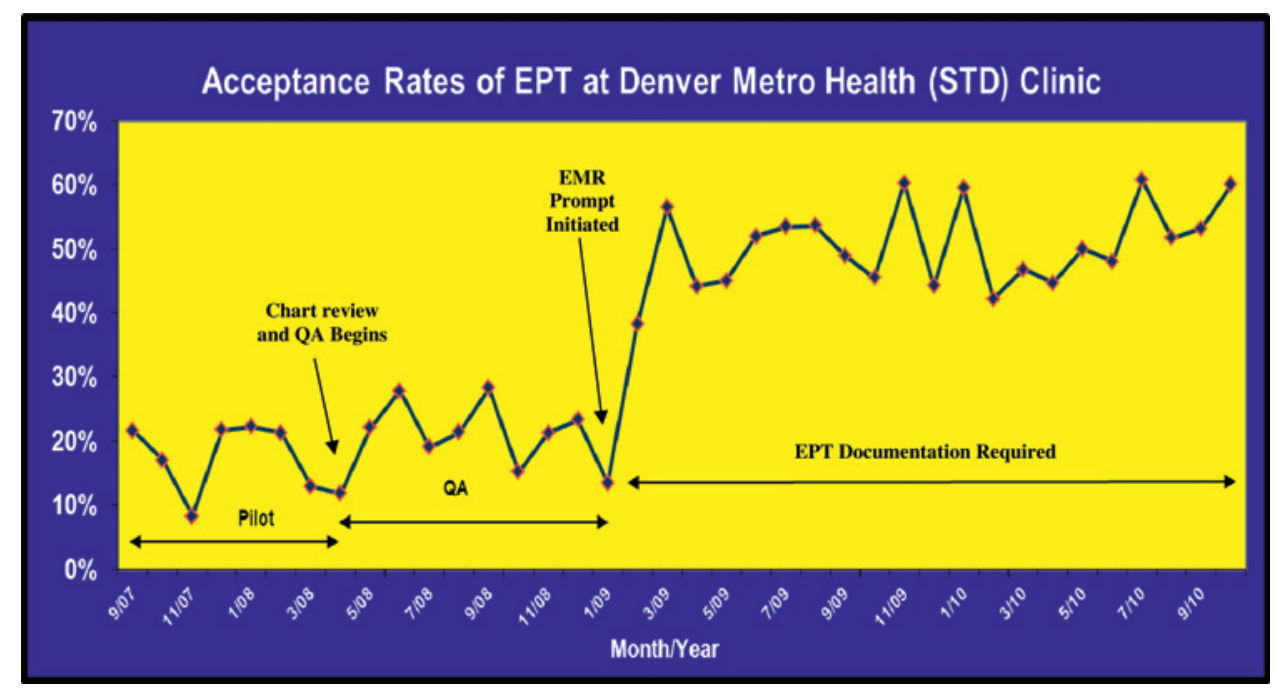

Abstract 05-S3.02 Figure 1 Acceptance rates of EPT Denver Metro Health (STD) clinic. 